\title{
The involvement of physico-chemical interactions in the adhesion of Candida albicans and Candida dubliniensis to epithelial cells
}

\author{
Mariana Henriques, Joana Azeredo and Rosário Oliveira \\ Centro de Engenharia Biológica - CEB, Universidade do Minho, Campus Gualtar, Braga, Portugal
}

\section{Summary}

\begin{abstract}
Candida albicans and Candida dubliniensis are two pathogenic yeasts particularly hazardous to immunocompromised patients. Adhesion of yeast cells to epithelium is considered one of the virulence factors and its study is of major importance. The main aim of this study was the comparison of the influence of physico-chemical properties on the adhesion of C. albicans and C. dubliniensis to epithelium. Two strains of each Candida species were used in the adhesion assays to HeLa cells. Adhered cells were enumerated by direct microscopic images observation. Yeast cell surface tension parameters and degree of hydrophobicity were determined by contact angle measurement. Pseudohyphae and hyphae formation was analysed by scanning electron microscopy. Yeast cells presented no statistical differences concerning their physicochemical surface properties. However, the extent of adhesion to epithelium was different among the four strains. As general conclusion, yeast adhesion to epithelium seems to be strain-dependent and not directly correlated with pseudohyphae formation.
\end{abstract}

Key words: Candida albicans, Candida dubliniensis, adhesion, epithelial cells.

\section{Introduction}

Candida species are fungal pathogens responsible for oral nosocomial infections. Candida albicans is the primary aetiological agent of oral candidiasis and on account of that has been largely phenotypically and genotypically studied. After being mismatched for years with C. albicans, in 1995 Candida dubliniensis was described as a new Candida species. ${ }^{1}$ Candida dubliniensis can cause disease independently of other Candida species, at least in HIV patients.

Colonisation of mucosal surfaces by pathogenic Candida species depends on their ability to adhere to such surfaces. Adhesion is, therefore, the first step in the process, leading to persistent colonisation and infection and the ability to adhere constitutes an important determinant of virulence. ${ }^{2}$

Some of the attributes of Candida species that are considered important virulence determinants include

Correspondence: Mariana Henriques, Department of Biological Engineering, University of Minho, Campus Gualtar, 4710-057 Braga, Portugal.

Tel.: +35125360 4400. Fax: +351253678986.

E-mail: mcrh@deb.uminho.pt

Accepted for publication 10 February 2007 the ability to form hyphae, ${ }^{3}$ to resist phagocytosis ${ }^{4}$ and to produce extracellular hydrolytic enzymes such as proteinases. $^{5}$

Adherence to host tissue is achieved by a combination of specific and non-specific mechanisms. Specific mechanisms include ligand-receptors interactions and non-specific mechanisms include electrostatic forces, aggregation and hydrophobic interactions. ${ }^{6}$

Epithelial cells, teeth and prosthetic devices are the oral cavity surfaces most prone to be colonised by Candida species.

In almost all studies concerning adhesion of oral micro-organisms to surfaces the saliva used is obtained from donors. ${ }^{7,8}$ Nevertheless, natural saliva varies according to the donor and the time of the day, thus exact duplications are impossible. Furthermore, natural saliva contains proteins such as mucin that can coat the oral surfaces influencing adhesion by specific interactions. Hence, artificial saliva can be used in order to focus only the physico-chemical interactions.

The objective of the present study was the comparison of the extent of adhesion between C. albicans and C. dubliniensis to epithelium. The influence of physicochemical yeast cells surface properties and pseudohyphae formation were the specific parameters evaluated. 


\section{Materials and methods}

\section{Yeast-growing conditions}

Two strains of C. albicans (C. albicans $12 \mathrm{~A}$ and C. albicans B311) and two strains of $C$. dubliniensis (C. dubliniensis CBS 7987 and $C$. dubliniensis CBS 7988) were used in this study. One of the strains of $C$. albicans is a clinical isolate, kindly provided by the Department of Biology of the University of Minho and the other C. albicans strain was obtained from the American Type Culture Collection (ATCC). The two C. dubliniensis were obtained from Centraalbureau voor Schimmelcultures (CBS). The yeast cells were subcultured in Sabouraud dextrose agar for $24 \mathrm{~h}$ and then grown in Sabouraud dextrose broth (SDB; VWR, Lisboa, Portugal) for $18 \mathrm{~h}$, at $37^{\circ} \mathrm{C}$ under agitation, until stationary phase. Cells were harvested by centrifugation $(2795 \mathrm{~g}$, for $10 \mathrm{~min})$ and washed twice with ultrapure water. The cells were enumerated using a hematocytometer (Mareinfeld GmbH \& Co KG, Lauda-Koenigshofen, Germany) and diluted in saline solution $(0.9 \% \mathrm{NaCl})$ or artificial saliva to the concentration needed for each assay.

\section{Epithelial cells}

The epithelial cells used were from a HeLa cell line gently provided by $\mathrm{Dr}^{\mathrm{a}}$ Elsa Anes from the Faculty of Pharmacy of the University of Lisbon. HeLa cells were cultured in DMEM (Sigma, Lisboa, Portugal) containing $10 \%$ of foetal bovine serum (Sigma), in cell culture flasks. After detachment, $10^{5}$ cells $\mathrm{ml}^{-1}$ were added to a 24-well plate containing circular lamellas $(\varphi=12 \mathrm{~mm})$ at the bottom. When the cells reached the confluence, they were washed two times with phosphate-buffered saline (PBS) and were used in the adhesion assays.

\section{Saliva preparation}

Artificial saliva was prepared according to Gal et al. [9] with the following composition in $\mathrm{mg} \mathrm{l}^{-1}: 125.6 \mathrm{NaCl}$, $963.9 \mathrm{KCl}, 189.2 \mathrm{KSCN}, 654.5 \mathrm{KH}_{2} \mathrm{PO}_{4}, 200.0$ Urea, $763.2 \mathrm{Na}_{2} \mathrm{SO}_{4} \cdot 10 \mathrm{H}_{2} \mathrm{O}, 178.0 \mathrm{NH}_{4} \mathrm{Cl}, 227.8 \mathrm{CaCl}_{2} \cdot 2 \mathrm{H}_{2} \mathrm{O}$ and $630.8 \mathrm{NaHCO}_{3}$. The $\mathrm{pH}$ was adjusted with carbon dioxide to 6.8 .

\section{Yeast cell surface properties}

Sample preparation.

Yeast cells were harvested by centrifugation at $2907 \mathrm{~g}$ for $10 \mathrm{~min}$ and washed with ultrapure water. The resulting pellet was resuspended in $100 \mathrm{ml}$ of saline solution or saliva to a concentration of $10^{9}$ cells $\mathrm{ml}^{-1}$. The suspension was filtered in a $3 \mu \mathrm{m}$ membrane under vacuum. Membranes were cut into three parts and dried in a Petri plate containing $20 \mathrm{~g} \mathrm{l}^{-1}$ of agar and $10 \%$ of glycerol for $2.5 \mathrm{~h}$.

Contact angle measurement.

Contact angles were measured by the sessile drop technique, on the cell lawns prepared previously, using an apparatus model OCA 15 PLUS, DATAPHYSICS (DataPhysics Instruments GmbH, Filderstadt, Germany). The measurements were performed, at room temperature, using three different liquids: water (VWR), formamide (VWR) and $\boldsymbol{\alpha}$-bromonaphthalene (VWR). Every assay was performed in triplicate and at least 10 contact angles, per sample, were measured.

\section{Adhesion assays}

Yeast cells were suspended to $10^{7}$ cells $\mathrm{ml}^{-1}$ (in saline solution or artificial saliva) and $1 \mathrm{ml}$ was added to each well, containing a glass lamella covered with a confluent layer of epithelial cells. After $1 \mathrm{~h}$ of incubation (100 rpm, at $37^{\circ} \mathrm{C}$ ) each well was washed twice with saline solution, by pipetting carefully only the liquid above the coupon. Finally, all the liquid was removed. The glass lamellas were withdrawn from the wells and were Gram-stained. The samples were observed microscopically and the images were captured on a computer. Twenty-five fields were randomly counted in each sample to determine the number of adhered cells. Each experiment was repeated three times.

\section{Statistical analysis}

The resulting data were statistically analysed using Statistical Package for the Social Sciences (SPSs). Oneway ANOvA with Bonferroni test was used to compare the number of adhered cells in the four strains. For the comparison between the two media (saline solution and artificial saliva) the independent $t$-test was used. All tests were performed with a confidence level of $95 \%$.

\section{Results}

The average number of yeast adhered to one epithelial cell in saline solution (used as control) and artificial saliva is illustrated in Fig. 1.

There was no significant difference in the number of adhered $C$. albicans $12 \mathrm{~A}$ and both strains of $C$. dubliniensis in the presence of saline solution $(P>0.05)$. In saliva, the number of adhered $C$. albicans $12 \mathrm{~A}$ and C. dubliniensis 7988 presented significant differences 


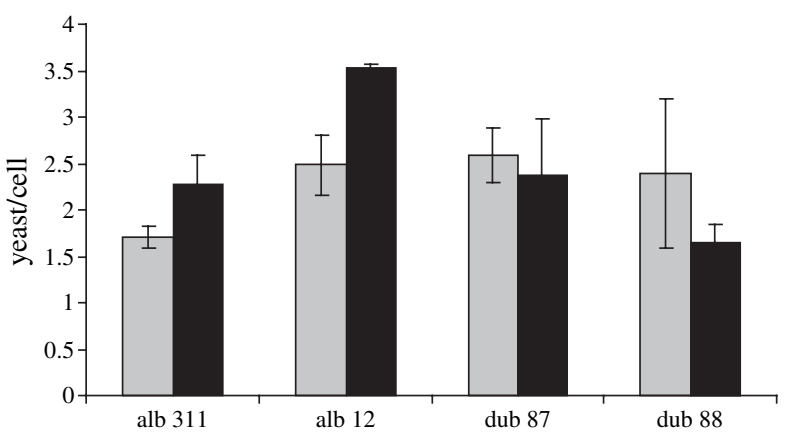

Figure 1 Average number of Candida albicans B311 (alb B311), C. albicans 12A (alb12), Candida dubliniensis 7987 (dub87) and C. dubliniensis 7988 (dub88) adhered to one epithelial cell in saline solution $(\square)$ or artificial saliva $(\square)$.

$(P<0.05)$, while the number of adhered $C$. albicans B311 and $C$. dubliniensis 7987 were not statistically different $(P>0.05)$. In saline solution, $C$. albicans B311 presented the lower number of adhered cells. However, in the presence of artificial saliva the lower number of yeast per epithelial cell was obtained for $C$. dubliniensis. Comparing the adherence behaviour in both media all the strains showed significant differences $(P<0.05)$. It is interesting to note that the number of both strains of C. albicans adhered to epithelium increased in the presence of artificial saliva, while the number of adhered C. dubliniensis decreased.

The percentage of HeLa cells without adhered Candida, with one and two or more yeast per cell was determined by direct enumeration of microscopic images (Fig. 2).

In the case of $C$. albicans $12 \mathrm{~A}$ there was a decrease in the percentage of HeLa cells without yeast and an increase in the percentage of HeLa cells with two or more yeast per cell, from saline solution to artificial saliva (Fig. 2). On the contrary, in artificial saliva, the number of HeLa cells without $C$. dubliniensis 7987 or C. dubliniensis 7988 increased while the number of HeLa cells with two or more yeast increased, these changes were more significant for the second strain. Comparing the number of $C$. albicans B311 adhered in the presence of saline solution or in artificial saliva, there was no alteration in the percentage of epithelial cells without yeast; the amount of HeLa cells with one yeast decreased and the number of HeLa cells with two or more yeast increased. The surface properties of the yeast strains are presented in Table 1.

The value of the free energy $\left(\Delta G_{\mathrm{ymy}}\right)$ represents the degree of hydrophobicity of the surface; if $\Delta G_{\text {ymy }}>0$ the surface can be considered as having a hydrophilic character and on the contrary, if $\Delta G_{\text {ymy }}<0$, the surface is hydrophobic. ${ }^{10}$ In the present study, all the yeast strains either in saline solution or artificial saliva, present a hydrophilic character (Table 1). However, $C$. albicans B311 presents a lower value of $\Delta G_{\mathrm{ymy}}$ in both media, meaning that this strain has a less hydrophilic character.

Hyphae formation is also an important factor that can be a determinant in the adhesion phenomenon (Fig. 3). Candida albicans B311 formed pseudohyphae either in saline solution or in artificial saliva (Fig. 3). The other strains formed hyphae only in the presence of artificial saliva.

The microscopic observations revealed that yeast cells adhered preferentially to the borders of the outer surface of epithelial cells. Scanning electron microscopic images of samples from the adhesion assays of C. albicans $12 \mathrm{~A}$ also corroborated this observation (Fig. 4).

\section{Discussion}

The most common in vitro models of mammalian cell lines used to study Candida infection, are exfoliated buccal epithelial cells (BECs), vaginal, urogenital and corneal cells. However, problems arise with exfoliated epithelial cell preparations, due to the presence of heterogeneous populations that show an abundance of non-viable cells, bacterial contamination and different degrees of enzymatic modifications of the cell surface. To avoid such problems, the use of uniform population of cells in measuring adherence has been more common. These include HeLa and human embryonic kidney
Figure 2 Percentage of epithelial cells without yeast adhered $(\square)$, with one yeast $(\square)$ or with two or more $(\square)$ in the presence of saline solution (a) or artificial saliva (b).
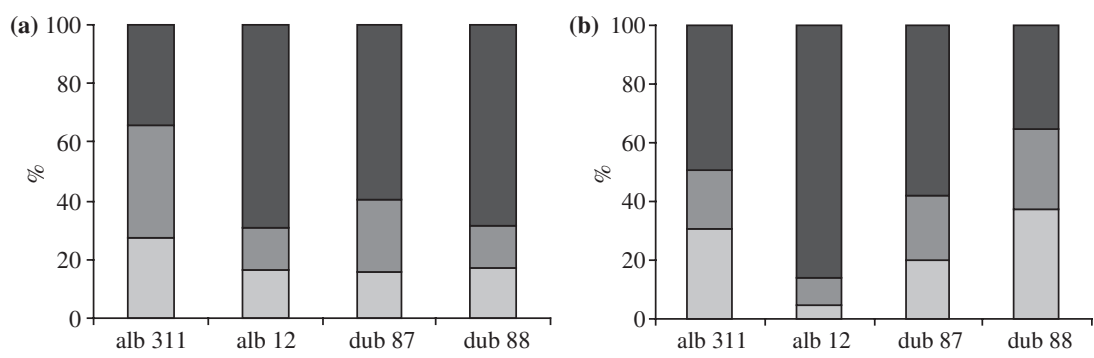
Table 1 Values of the surface tension parameters $\left(\gamma^{+}, \gamma^{-}, \gamma^{\mathrm{LW}}\right)$ and the free energy of interaction $\left(\Delta G_{\mathrm{ymy}}\right)$ between two identical yeast cells (y) immersed in medium (m) of Candida albicans B311 (albB311), C. albicans 12A (alb12), Candida dubliniensis 7987 (dub87) and C. dubliniensis 7988 (dub88) determined in saline solution and in artificial saliva

\begin{tabular}{llllll}
\hline Medium & Cells & $\gamma^{+}\left(\mathrm{mJ} \mathrm{m}^{-2} ; \pm \mathrm{SD}\right)$ & $\gamma^{-}\left(\mathrm{mJ} \mathrm{m}^{-2} ; \pm \mathrm{SD}\right)$ & $\gamma^{\mathrm{LW}}\left(\mathrm{mJ} \mathrm{m}^{-2} ; \pm \mathrm{SD}\right)$ & $\Delta G_{\mathrm{sws}}\left(\mathrm{mJ} \mathrm{m} \mathrm{m}^{-2} ; \pm \mathrm{SD}\right)$ \\
\hline Saline & C. albicans B311 & $3 \pm 1$ & $46 \pm 2$ & $29 \pm 1$ & $21 \pm 1$ \\
Solution & C. albicans 12A & $4 \pm 1$ & $51 \pm 3$ & $27 \pm 5$ & $25 \pm 5$ \\
& C. dubliniensis 7987 & $3 \pm 0$ & $52 \pm 1$ & $30 \pm 0$ & $27 \pm 1$ \\
& C. dubliniensis 7988 & $4 \pm 2$ & $40 \pm 11$ & $39 \pm 9$ & $24 \pm 6$ \\
Artificial & C. albicans B311 & $6 \pm 1$ & $51 \pm 1$ & $23 \pm 1$ & $21 \pm 4$ \\
Saliva & C. albicans 12A & $3 \pm 0$ & $49 \pm 3$ & $24 \pm 1$ & $24 \pm 2$ \\
& C. dubliniensis 7987 & $6 \pm 1$ & $52 \pm 0$ & $25 \pm 3$ & $22 \pm 2$ \\
& C. dubliniensis 7988 & $6 \pm 2$ & $53 \pm 1$ & & $23 \pm 4$ \\
\hline
\end{tabular}
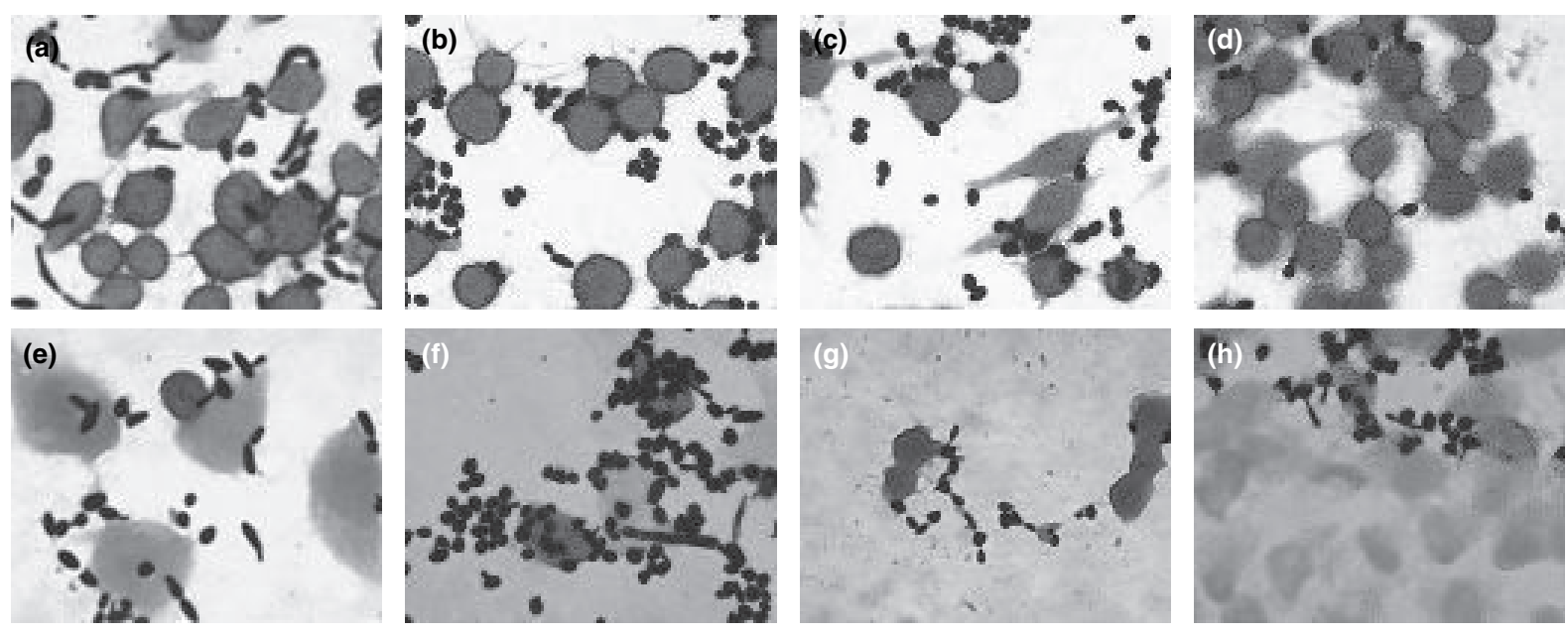

Figure 3 Images of Candida albicans B311 (a and e), of C. albicans 12A (b and f), Candida dubliniensis 7987 (c and g) and C. dubliniensis 7988 $(\mathrm{d}$ and $\mathrm{h})$ adhered to epithelium in saline solution (a-d) or artificial saliva (e-h).
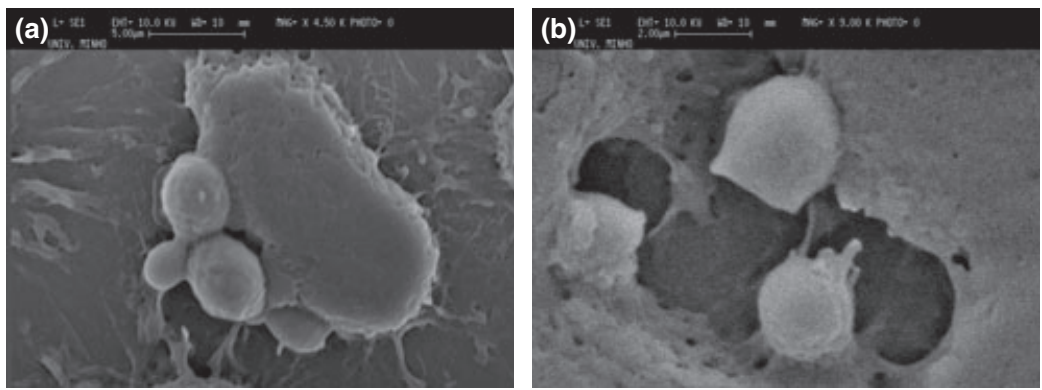

Figure 4 Images of Candida albicans 12A adhered to epithelial cells observed by scanning electron microscope with $4500 \times$ (a) and $9000 \times$ (b) of magnification. epithelial cells, fibroblasts and HEp- 2 cells. ${ }^{6}$ A HeLa cell line was used in this study and all Candida strains presented high levels of adherence to this cellular line. The extent of yeast cell adhesion in saline solution, evaluated by the number of yeast attached to each HeLa cell was very similar for all strains. Conversely, when the adhesion medium was artificial saliva, C. albicans $12 \mathrm{~A}$ adhered in a significantly larger extent than C. dubliniensis 7988, although C. albicans B311 and C. dubliniensis 7987 presented no differences. Vidotto et al. [11] studied the adhesion of $C$. albicans and C. dubliniensis to $\mathrm{BEC}$ and vaginal epithelial cells in the presence of PBS and they found differences in the adhesion behaviours, being $C$. albicans the strain that presented a higher extent of adhesion. However, Gilfilland et al. [12] showed that oral $C$. dubliniensis isolates were more adherent to BEC than C. albicans when grown in glucose and equally adherent when grown in galactose. Nevertheless, according to several authors, ${ }^{13-15}$ the greater extent of adhesion of some strains of $C$. albicans with 
respect to $C$. dubliniensis to $\mathrm{BEC}$ and vaginal epithelial cells is in agreement with the fact that $C$. albicans is usually considered more virulent than C. dubliniensis. ${ }^{11}$

The adhesion of these strains to acrylic and hydroxyapatite (HAP) was also studied ${ }^{16}$ and no differences among the strains were encountered, either in water or in saliva as well. This similar behaviour was explained by the similar surface properties (zeta potential, surface tension and hydrophilicity) of the four strains. It is well documented that the adhesion phenomenon to inert surfaces is ruled by physico-chemical properties of microbial cell surfaces. ${ }^{17,18}$ It is not clear, however, if these properties also determine microbial adhesion to human epithelium.

The values of yeast cell surface tension and hydrophobicity (Table 1) for all the strains studied are statistically similar and so these properties are not able to differentiate among the strains' ability to adhere to epithelium. Hence, as in the case of adhesion to inert surfaces, hydrophobic properties do not seem to rule adhesion. The electron donor parameters were found to be responsible for the adhesion phenomenon to inert surfaces. ${ }^{16}$ However, this was not evident in adhesion to epithelium. It must be stressed that the extent of yeast adhesion to epithelial cells can only be compared with that to inert surfaces in a qualitative way, as in the first case it was the number of yeast per epithelial cells that was determined, while on the inert surfaces it was the number of yeast per $\mathrm{mm}^{2}$ that was quantified. There are some authors ${ }^{19}$ who have measured the number of yeast per $\mathrm{mm}^{2}$ in the case of adhesion to epithelial cells, although this is only possible if the cells are 100\% confluent, which is difficult to achieve.

In the present study, the number of cells of both strains of C. albicans adhered to HeLa cells increased in the presence of artificial saliva, while the contrary happened for C. dubliniensis strains.

The role of saliva in the adhesion to epithelium has been largely studied in the case of C. albicans. Although some authors ${ }^{20}$ found that saliva promotes the adhesion of C. albicans to epithelial cells, others ${ }^{21}$ report the opposite. Indeed, the influence of saliva in adhesion depends on various factors, such as the origin and composition and also on the strain of Candida that is being studied.

The factors affecting Candida adhesion to epithelial cells can depend on the yeast, on the epithelial cells or on environmental factors. Within yeast factors can be included cell concentration and viability, the growth phase and temperature, the growth medium composition, species and strains and germ tube formation. $^{2}$
Candida albicans and C. dubliniensis are the two Candida strains with a capacity to form true hyphae in addition to pseudohyphae. ${ }^{22}$ The transition from yeast to hyphae form is one factor of Candida virulence. Hyphae formation depends on the medium used to grow the yeast cells and the number of formed hyphae increases with time. ${ }^{12}$ In this study, C. albicans B311 presented hyphal formation in both adhesion media, saline solution and artificial saliva. While the other strains formed hyphae only in the presence of artificial saliva.

Although the cells were grown in SDB and put into contact with artificial saliva only during the adhesion assay $(1 \mathrm{~h})$, all strains studied presented hyphal formation in this case. Other authors ${ }^{12}$ also found that hyphae formation can occur after $1 \mathrm{~h}$ either for C. albicans or C. dubliniensis in different media.

The environmental factors that favour germination, formation of pseudohyphae or hyphae, include temperature higher than $35^{\circ} \mathrm{C}$ and $\mathrm{pH}$ of $6.5-7 .{ }^{22} \mathrm{The} \mathrm{pH}$ of the artificial saliva used was 6.8 that can explain the formation of hyphae by all the strains.

According to Nikawa et al. [23], in some C. albicans strains the adhesion capability increases in the presence of germ tubes when compared with blastopores. However, for other strains no significant differences were noticed.

Hyphal formation did not seem to play an important role in the adhesion of Candida. Hence, other factors, rather than physico-chemical properties or hyphal formation seem to be ruling the process of adhesion. Among these factors are the peripheral proteins that promote adhesion, called adhesins. A number of proteins have been identified that recognise host cell ligands, including MP60, MP58, MP66, MP130 and MP37. ${ }^{24}$

Ultrastructural evidence indicates that specific interactions between Candida and epithelial cells are mediated by a flocular-fibrillar adhesin layer present on the outer surface of the yeast. ${ }^{25}$ The Candida surface is enriched with concavalin A-binding sites and attachment to the epithelial cells is mediated by fibrillar structures or polysaccharide granules distributed on the cell wall coat. ${ }^{25}$

Candida albicans and C. dubliniensis have equal abilities to adhere to inert oral surfaces ${ }^{16}$ and the adhesion is enhanced in the presence of artificial saliva due to an increase in the physico-chemical interactions. Considering adhesion to epithelium, other factors rather than physico-chemical ones seem to rule the phenomenon. Additionally differences in the adhesion capabilities were clearly shown among the four strains. Hence, adhesion to epithelium is strain-dependent, conversely to adhesion to inert surfaces. 


\section{Acknowledgments}

The authors fully acknowledge the financial support of FCT/Portugal through the grant BD3195/2000 and the project POCTI/BIO/42638/2001. We also would like to thank Dr Célia Pais and Dr Judite Almeida from the Department of Biology, University of Minho, for providing the Candida albicans 12A strain, Dr Manuel Vilanova from Instituto de Ciêncicas Biomédicas Abel Salazar, University of Porto, for providing C. albicans B311 and Dr Elsa Anes from Faculty of Pharmacy, University of Lisbon, for providing the HeLa cell line.

\section{References}

1 Sullivan DJ, Westerneng TJ, Haynes KA, Bennett DE, Coleman DC. Candida dubliniensis sp. nov.: phenotypic and molecular characterisation of a novel species associated with oral candidosis in HIV-infected individuals. Microbiology 1995; 141: 1507-21.

2 Douglas J. Adhesion of Candida species to epithelial surfaces. Crit Rev Microbiol 1987; 15: 27-43.

3 Sobel JD, Muller G, Buckley HR. Critical role of germ tube formation in the pathogenesis of candidal vaginitis. Infect Immun 1984; 44: 576-80.

4 Stanley VC, Hurley R. The growth of Candida species in cultures of mouse peritoneal macrophages. J Pathol 1969; 97: 357-66.

5 Odds FC. Candida albicans proteinase as a virulence factor in the pathogenesis of Candida infections. Zentralbl Bakteriol Parasitenkd Infektionskr Hyg Abt 1985; 260: 539-42.

6 Cotter G, Kavanagh K. Adherence mechanisms of Candida albicans. Br J Biomed Sci 2000; 57: 241-9.

7 Ueta E, Tanida T, Doi S, Osaki T. Regulation of Candida albicans growth and adhesion by saliva. J Lab Clin Med 2000; 136: 67-73.

8 Nikawa H, Chen J, Hamada T, Nishimura M, Polyzois G. Candida albicans colonization on thermal cycled maxillofacial polymeric materials in vitro. J Oral Rehabil 2001; 28: 526-33.

9 Gal J, Fovet Y, Adib-Yadzi M. About a synthetic saliva for in vitro studies. Talanta 2001; 53: 1103-15.

10 van Oss CJ, Giese RF. The hydrophilicity and hydrophobicity of clay minerals. Clay Miner 1995; 2: 347-69.

11 Vidotto V, Mantoan B, Pugliese A. et al. Adherence of Candida albicans and Candida dubliniensis to buccal and vaginal cells. Rev Iber Micol 2003; 20: 52-4.
12 Gilfillan GD, Sullivan DJ, Haynes K, Parkinson T, Coleman DC, Gow NAR. Candida dubliniensis: phylogeny and putative virulence factors. Microbiology 1998; 144: 829-38.

13 Gale CA, Bendel CM, McClellan M, Becker JM, Berman J, Hostetter MK. Linkage of adhesion, filamentous growth and virulence in Candida albicans to a single gene, INTI. Science 1998; 279: 1355-8.

14 Pereiro M, Losada A, Toribio J. Adherence of Candida albicans strains isolated from AIDS patients. Comparison with pathogenic yeasts isolated from patients without HIV infection. Br J Dermatol 1997; 137: 76-80.

15 Calderone R, Suzuki S, Cannon RD. Candida albicans: adherence, signaling and virulence. Med Mycol 2000; 38 : 125-37.

16 Henriques M, Azeredo J, Oliveira R. Adhesion of Candida albicans and Candida dubliniensis to acrylic and hydroxipapatite. Colloids Surf B Biointerfaces 2004; 33: 235-41.

17 Millsap KW, Bos R, Busscher HJ, van der Mei HC. Surface aggregation of Candida albicans on glass in the absence and presence of adhering Streptococcus gordonii in a parallelplate flow chamber: a surface thermodynamical analysis based on acid-base interactions. J Colloid Interface Sci 1999; 212: 495-502.

18 Rotrosen D, Calderone RA, Edwards JE. Adherence of Candida species to host tissues and plastic surfaces. Rev Inf Dis 1986; 8: 73-85.

19 Dorocka-Bobkowska B, Konopka K, Duzgunes N. Influence of antifungal polyenes on the adhesion of Candida albicans and Candida glabarata to human epithelial cells in vitro. Arch Oral Biol 2003; 48: 805-15.

20 Holmes AD, Bandara BMK, Cannon RD. Saliva promotes Candida albicans adherence to human epithelial cells. J Dent Res 2002; 81: 28-32.

21 Umazume M, Ueta E, Osaki T. Reduced inhibition of Candida albicans adhesion by saliva from patients receiving oral cancer therapy. J Clin Microbiol 1995; 33: 432-9.

22 Calderone R. Taxonomy and biology of Candida. In: Calderone R (ed.), Candida and Candidiasis. Washington: ASM Press, 2004: 15-27.

23 Nikawa H, Egusa H, Makihira S. et al. A novel technique to evaluate the ahdesion of Candida species to gingival epithelial cells. Mycoses 2002; 46: 384-9.

24 Sturtevant J, Calderone R. Candida albicans adhesins: biochemical aspects and virulence. Rev Iber Micol 1997; 14: 90-7.

25 Ghannoum MA, Radwan SS. Candida Adherence to Epithelial Cells. Kuweit: CRC Press, 1990. 